International Journal of Pure and Applied Mathematics

Volume 89 No. 1 2013, 41-53

ISSN: 1311-8080 (printed version); ISSN: 1314-3395 (on-line version)

url: http://www.ijpam.eu

doi: http://dx.doi.org/10.12732/ijpam.v89i1.6

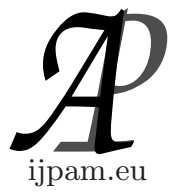

\title{
RIGHT RESIDUATED LATTICES AND RIGHT TRIANGLE ALGEBRAS
}

\author{
Yong Chan Kim \\ Department of Mathematics \\ Gangneung-Wonju University \\ Gangneung, Gangwondo, 210-702, KOREA
}

\begin{abstract}
In this paper, we introduce the notions of right (left) triangle algebras in right (left) residuated lattices. Every right (left) residuated lattices induce right (left) triangle algebras. Moreover, every right (left) triangle algebras are isomorphic their right (left) approximation spaces.
\end{abstract}

AMS Subject Classification: 03E72, 54A40, 54B10

Key Words: right (left) residuated lattices, right (left) rough approximation operators, right (left) triangle algebras

\section{Introduction}

Pawlak $[10,11]$ introduced rough set theory as a formal tool to deal with imprecision and uncertainty in data analysis. Hájek [8] introduced a complete residuated lattice which is an algebraic structure for many valued logic. Moreover, Georgescu and Popescue [6,7] introduced a generalized residuated lattice which is induced by two implications. By using these concepts, information systems and decision rules are investigated $[2,9,14]$. Deschrijver, et.al. [3-5,12,13] introduced triangle algebras and interval-valued residuated lattices.

In this paper, we introduce the notions of right (left) triangle algebras in right (left) residuated lattices. Every right (left) residuated lattices induce

Received: June 15, 2013

(c) 2013 Academic Publications, Ltd. url: www.acadpubl.eu 
right (left) triangle algebras. Moreover, every right (left) triangle algebras are isomorphic their right (left) approximation spaces.

Definition 1. (see [6], [7]) A structure $(L, \vee, \wedge, \odot, \Rightarrow, \perp, \top)$ is called a right residuated lattice if it satisfies the following conditions:

(R1) $(L, \vee, \wedge, \top, \perp)$ is a bounded where $\perp$ is the bottom element and $\perp$ is the top element;

(R2) $(L, \odot, \top)$ is a monoid;

$(\mathrm{RR})$ it satisfies a right residuation, i.e.

$$
a \odot b \leq c \text { iff } b \leq a \Rightarrow c
$$

A structure $(L, \vee, \wedge, \odot, \rightarrow, \perp, \top)$ is called a left residuated lattice if it satisfies (R1), (R2) and

(LR) it satisfies a left residuation, i.e.

$$
a \odot b \leq c \text { iff } a \leq b \rightarrow c .
$$

A structure $(L, \vee, \wedge, \odot, \Rightarrow, \rightarrow, \perp, \top)$ is called a generalized residuated lattice if it is a right and left residuated lattice.

We call that a generalized residuated lattice has the law of double negation if $a=\left(a^{*}\right)^{0}=\left(a^{0}\right)^{*}$ where $a^{0}=a \rightarrow \perp$ and $a^{*}=a \Rightarrow \perp$.

Remark 2. (see [6], [7], [12] (1) A generalized residuated lattice is a residuated lattice $(\rightarrow=\Rightarrow)$ iff $\odot$ is commutative.

(2) A left-continuous t-norm $([0,1], \leq, \odot)$ defined by $a \rightarrow b=\bigvee\{c \mid a \odot c \leq$ $b\}$ is a residuated lattice

(3) A pseudo MV-algebra is a generalized residuated lattice with the law of double negation.

Lemma 3. (see [4], [5]) Let $(L, \wedge, \vee, \odot, \rightarrow, \Rightarrow, \perp, \top)$ be a generalized residuated lattice. For each $x, y, z, x_{i}, y_{i} \in L$, we have the following properties.

(1) If $y \leq z,(x \odot y) \leq(x \odot z), x \rightarrow y \leq x \rightarrow z$ and $z \rightarrow x \leq y \rightarrow x$ for $\rightarrow \in\{\rightarrow, \Rightarrow\}$.

(2) $x \odot y \leq x \wedge y$.

$(3) x \rightarrow\left(\bigwedge_{i \in \Gamma} y_{i}\right)=\bigwedge_{i \in \Gamma}\left(x \rightarrow y_{i}\right)$ and $\left(\bigvee_{i \in \Gamma} x_{i}\right) \rightarrow y=\bigwedge_{i \in \Gamma}\left(x_{i} \rightarrow y\right)$ for $\rightarrow \in\{\rightarrow, \Rightarrow\}$. 
(4) $(x \odot y) \rightarrow z=x \rightarrow(y \rightarrow z)$ and $(x \odot y) \Rightarrow z=y \Rightarrow(x \Rightarrow z)$.

(5) $x \rightarrow(y \Rightarrow z)=y \Rightarrow(x \rightarrow z)$ and $x \Rightarrow(y \rightarrow z)=y \rightarrow(x \Rightarrow z)$.

(6) $x \odot(x \rightarrow y) \leq y$ and $(x \Rightarrow y) \odot x \leq y$.

(7) $(x \Rightarrow y) \odot(y \Rightarrow z) \leq x \Rightarrow z$ and $(y \rightarrow z) \odot(x \rightarrow y) \leq x \rightarrow z$.

(8) $x \rightarrow y=\top$ iff $x \leq y$ iff $x \Rightarrow y=\top$.

Definition 4. A structure $\mathcal{A}=\left(A, \wedge, \vee, \odot, \Rightarrow, \nu_{1}, \mu_{1}, \perp, e_{1}, \top\right)$ is called a right triangle algebra if it satisfies the following conditions:

(R) $(A, \wedge, \vee, \odot, \Rightarrow, \perp, \top)$ is a right residuated lattice.

(T1) $\nu_{1}(x) \leq x$ and $\nu_{1}(x) \leq \nu_{1}\left(\nu_{1}(x)\right)$.

(T2) $\nu_{1}(x \wedge y)=\nu_{1}(x) \wedge \nu_{1}(y)$ and $\nu_{1}(x \vee y)=\nu_{1}(x) \vee \nu_{1}(y)$.

(T3) $\nu_{1}\left(e_{1}\right)=\perp$.

(T4) $\nu_{1} \circ \mu_{1}=\mu_{1}$.

(S1) $x \leq \mu_{1}(x)$ and $\mu_{1}(x) \geq \mu_{1}\left(\mu_{1}(x)\right)$.

(S2) $\mu_{1}(x \wedge y)=\mu_{1}(x) \wedge \mu_{1}(y)$ and $\mu_{1}(x \vee y)=\mu_{1}(x) \vee \mu_{1}(y)$.

(S3) $\mu_{1}\left(e_{1}\right)=\top$.

(S4) $\mu_{1} \circ \nu_{1}=\nu_{1}$.

(R5) $\nu_{1}(x \Rightarrow y) \leq \nu_{1}(x) \Rightarrow \nu_{1}(y)$.

(R6) $\left(\nu_{1}(x) \Leftrightarrow \nu_{1}(y)\right) \odot\left(\mu_{1}(x) \Leftrightarrow \mu_{1}(y)\right) \leq(x \Leftrightarrow y)$.

(R7) $\nu_{1}(x) \Rightarrow \nu_{1}(y) \leq \nu_{1}\left(\nu_{1}(x) \Rightarrow \nu_{1}(y)\right)$.

The pair $\left[\nu_{1}(x), \mu_{1}(x)\right]$ is called a right rough approximation for $x \in A$. $R_{r}(A)=\left\{\left[\nu_{1}(x), \mu_{1}(x)\right] \mid x \in A\right\}$ is called a right rough approximation space.

A structure $\mathcal{A}=\left(A, \wedge, \vee, \odot, \rightarrow, \nu_{2}, \mu_{2}, \perp, e_{2}, \top\right)$ is called a left triangle algebra if $\nu_{2}, \mu_{2}$ satisfy (T1)-(T4), (S1)-(S4), respectively and following conditions:

(L) $(A, \wedge, \vee, \odot, \rightarrow, \perp, \top)$ is a left residuated lattice.

(L5) $\nu_{2}(x \rightarrow y) \leq \nu_{2}(x) \rightarrow \nu_{2}(x)$.

(L6) $\left(\mu_{2}(x) \leftrightarrow \mu_{2}(y)\right) \odot\left(\nu_{2}(x) \leftrightarrow \nu_{2}(y)\right) \leq(x \leftrightarrow y)$.

$(\mathrm{L} 7) \nu_{2}(x) \rightarrow \nu_{2}(y) \leq \nu_{2}\left(\nu_{2}(x) \rightarrow \nu_{2}(y)\right)$.

The pair $\left[\nu_{2}(x), \mu_{2}(x)\right]$ is called a left rough approximation for $x \in A$. $R_{l}(A)=\left\{\left[\nu_{2}(x), \mu_{2}(x)\right] \mid x \in A\right\}$ is called a left rough approximation space. 
Remark 5. (1) If $\odot$ is commutative (or $\Rightarrow=\rightarrow$ ), $\nu_{1}=\nu_{2}, \mu_{1}=\mu_{2}$ and $e_{1}=e_{2}$ in Definition 4 , then $\left(A, \wedge, \vee, \odot, \Rightarrow, \nu_{1}, \mu_{1}, \perp, e_{1}, \top\right)$ is a triangle algebra in $[12]$.

(2) In Definition 4, $\nu_{i}(\top)=\top$ and $\mu_{i}(\perp)=\perp$ because $\nu_{i}(\top)=\nu_{i}\left(\mu_{i}\left(e_{i}\right)\right)=$ $\mu_{i}\left(e_{i}\right)=\top$ and $\mu_{i}(\perp)=\mu_{i}\left(\nu_{i}\left(e_{i}\right)\right)=\nu_{i}\left(e_{i}\right)=\perp$.

\section{Right Residuated Lattices and Right Triangle Algebras}

Theorem 6. Let $(A, \wedge, \vee, \odot, \Rightarrow, \perp, \top)$ be a right residuated lattice and $[A]^{[2]}=\{[x, y] \mid x \leq y\}$. Define

$$
\begin{aligned}
& {\left[x_{1}, x_{2}\right] \wedge\left[y_{1}, y_{2}\right]=\left[x_{1} \wedge y_{1}, x_{2} \wedge y_{2}\right]} \\
& {\left[x_{1}, x_{2}\right] \vee\left[y_{1}, y_{2}\right]=\left[x_{1} \vee y_{1}, x_{2} \vee y_{2}\right]} \\
& {\left[x_{1}, x_{2}\right] \odot\left[y_{1}, y_{2}\right]=\left[x_{1} \odot y_{1}, x_{2} \odot y_{2}\right]} \\
& {\left[x_{1}, x_{2}\right] \Rightarrow\left[y_{1}, y_{2}\right]=\left[\left(x_{1} \Rightarrow y_{1}\right) \wedge\left(x_{2} \Rightarrow y_{2}\right), x_{2} \Rightarrow y_{2}\right] .}
\end{aligned}
$$

Then the following properties hold.

(1) $\left([A]^{[2]}, \wedge, \vee, \odot, \Rightarrow,[\perp, \perp],[\top, \top]\right)$ is a right residuated lattice.

(2) $(D(A), \wedge, \vee, \odot, \Rightarrow,[\perp, \perp],[\top, \top])$ is a right residuated lattice where $D(A)$ $=\{[x, x] \mid x \in A\}$. Moreover, $\eta_{1}: A \rightarrow D(A)$ with $\eta_{1}(x)=[x, x]$ is an isomorphism;i.e, $\eta_{1}$ is bijective, $\eta_{1}(x \vee y)=\eta_{1}(x) \vee \eta_{1}(y), \eta_{1}(x \wedge y)=\eta_{1}(x) \wedge$ $\eta_{1}(y), \eta_{1}(x \odot y)=\eta_{1}(x) \odot \eta_{1}(y), \eta_{1}(x \Rightarrow y)=\eta_{1}(x) \Rightarrow \eta_{1}(y)$.

(3) If we define $\pi_{1}\left(\left[x_{1}, x_{2}\right]\right)=\left[x_{1}, x_{1}\right]$ and $\pi_{2}\left(\left[x_{1}, x_{2}\right]\right)=\left[x_{2}, x_{2}\right]$, then $\left([A]^{[2]}, \wedge, \vee, \odot, \Rightarrow, \pi_{1}, \pi_{2},[\perp, \perp],[\perp, \top],[\top, \top]\right)$ is a right triangle algebra.

Proof. (1)

$$
\begin{aligned}
& {\left[x_{1}, x_{2}\right] \odot\left[y_{1}, y_{2}\right]=\left[x_{1} \odot y_{1}, x_{2} \odot y_{2}\right] \leq\left[z_{1}, z_{2}\right]} \\
& \text { iff } x_{1} \odot y_{1} \leq z_{1}, x_{2} \odot y_{2} \leq z_{2} \text { iff } y_{1} \leq x_{1} \Rightarrow z_{1}, y_{2} \leq x_{2} \Rightarrow z_{2} \\
& \quad \text { iff }\left[y_{1}, y_{2}\right] \leq\left[\left(x_{1} \Rightarrow z_{1}\right) \wedge\left(x_{2} \Rightarrow z_{2}\right), x_{2} \Rightarrow z_{2}\right]=\left[x_{1}, x_{2}\right] \Rightarrow\left[z_{1}, z_{2}\right]
\end{aligned}
$$

Other cases are easily proved.

(2) $\eta_{1}(x \Rightarrow y)=[x \Rightarrow y, x \Rightarrow y]=[x, x] \Rightarrow[y, y]=\eta_{1}(x) \Rightarrow \eta_{1}(y)$. Other cases are easily proved.

(3) $(\mathrm{R} 5) \pi_{1}\left(\left[x_{1}, x_{2}\right] \Rightarrow\left[y_{1}, y_{2}\right]\right)=\left[\left(x_{1} \Rightarrow y_{1}\right) \wedge\left(x_{2} \Rightarrow y_{2}\right),\left(x_{1} \Rightarrow y_{1}\right) \wedge\left(x_{2} \Rightarrow\right.\right.$ $\left.\left.y_{2}\right)\right] \leq\left[\left(x_{1} \Rightarrow y_{1}\right),\left(x_{1} \Rightarrow y_{1}\right)\right]=\pi_{1}\left(\left[x_{1}, x_{2}\right]\right) \Rightarrow \pi_{1}\left(\left[y_{1}, y_{2}\right]\right)$. 
$(\mathrm{R} 6)$

$$
\begin{aligned}
& \pi_{1}\left(\left[x_{1}, x_{2}\right]\right) \Leftrightarrow \pi_{1}\left(\left[y_{1}, y_{2}\right]\right) \odot \pi_{2}\left(\left[x_{1}, x_{2}\right]\right) \Leftrightarrow \pi_{2}\left(\left[y_{1}, y_{2}\right]\right) \\
& =\left(\left[x_{1}, x_{1}\right] \Leftrightarrow\left[y_{1}, y_{1}\right]\right) \odot\left(\left[x_{2}, x_{2}\right] \Leftrightarrow\left[y_{2}, y_{2}\right]\right) \\
& =\left(\left[x_{1} \Leftrightarrow y_{1}, x_{1} \Leftrightarrow y_{1}\right]\right) \odot\left(\left[x_{2} \Leftrightarrow y_{2}, x_{2} \Leftrightarrow y_{2}\right]\right) \\
& =\left(\left[\left(x_{1} \Leftrightarrow y_{1}\right) \odot\left(x_{2} \Leftrightarrow y_{2}\right),\left(x_{1} \Leftrightarrow y_{1}\right) \odot\left(x_{2} \Leftrightarrow y_{2}\right)\right)\right. \\
& \leq\left(\left[\left(x_{1} \Leftrightarrow y_{1}\right) \wedge\left(x_{2} \Leftrightarrow y_{2}\right),\left(x_{2} \Leftrightarrow y_{2}\right)\right)\right. \\
& =\left[x_{1}, x_{2}\right] \Leftrightarrow\left[y_{1}, y_{2}\right] .
\end{aligned}
$$

(R7)

$$
\begin{aligned}
& \pi_{1}\left(\left[x_{1}, x_{2}\right]\right) \Rightarrow \pi_{1}\left(\left[y_{1}, y_{2}\right]\right)=\left[x_{1}, x_{1}\right] \Rightarrow\left[y_{1}, y_{1}\right] \\
& =\left[x_{1} \Rightarrow y_{1}, x_{1} \Rightarrow y_{1}\right]=\pi_{1}\left(\pi_{1}\left(\left[x_{1}, x_{2}\right]\right) \Rightarrow \pi_{1}\left(\left[y_{1}, y_{2}\right]\right)\right) .
\end{aligned}
$$

Hence $\left([A]^{[2]}, \wedge, \vee, \odot, \Rightarrow, \pi_{1}, \pi_{2},[\perp, \perp],[\perp, \top],[\top, \top]\right)$ is a right triangle algebra.

The following corollary is similarly proved that of Theorem 6 .

Corollary 7. Let $(A, \wedge, \vee, \odot, \rightarrow, \perp, \top)$ be a left residuated lattice and $[A]^{[2]}=\{[x, y] \mid x \leq y\}$. Define

$$
\begin{aligned}
& {\left[x_{1}, x_{2}\right] \wedge\left[y_{1}, y_{2}\right]=\left[x_{1} \wedge y_{1}, x_{2} \wedge y_{2}\right]} \\
& {\left[x_{1}, x_{2}\right] \vee\left[y_{1}, y_{2}\right]=\left[x_{1} \vee y_{1}, x_{2} \vee y_{2}\right]} \\
& {\left[x_{1}, x_{2}\right] \odot\left[y_{1}, y_{2}\right]=\left[x_{1} \odot y_{1}, x_{2} \odot y_{2}\right]} \\
& {\left[x_{1}, x_{2}\right] \rightarrow\left[x_{1}, x_{2}\right]=\left[\left(x_{1} \rightarrow y_{1}\right) \wedge\left(x_{2} \rightarrow y_{2}\right), x_{2} \rightarrow y_{2}\right]}
\end{aligned}
$$

Then the following properties hold.

(1) $\left([A]^{[2]}, \wedge, \vee, \odot, \rightarrow,[\perp, \perp],[\top, \top]\right)$ is a left residuated lattice.

(2) $(D(A), \wedge, \vee, \odot, \rightarrow,[\perp, \perp],[\top, \top])$ is a left residuated lattice where $D(A)=$ $\{[x, x] \mid x \in A\}$. Moreover, $\eta_{2}: A \rightarrow D(A)$ with $\eta_{2}(x)=[x, x]$ is an isomorphism in a sense in Theorem 6(2).

(3) If we define $\pi_{1}\left(\left[x_{1}, x_{2}\right]\right)=\left[x_{1}, x_{1}\right]$ and $\pi_{2}\left(\left[x_{1}, x_{2}\right]\right)=\left[x_{2}, x_{2}\right]$, then $\left([A]^{[2]}, \wedge, \vee, \odot, \rightarrow, \pi_{1}, \pi_{2},[\perp, \perp],[\perp, \top],[\top, \top]\right)$ is a left triangle algebra.

Theorem 8. Let $\left(A, \wedge, \vee, \otimes, \Rightarrow, \nu_{1}, \mu_{1}, \perp, e_{1}, \top\right)$ be a right triangle lattice.

Then the following properties hold:

(1) $\left(E_{r}(A), \wedge, \vee, \otimes, \Rightarrow, \perp, \top\right)$ is a right residuated lattice where $E_{r}(A)=$ $\left\{x \in A \mid \nu_{1}(x)=x\right\}=\left\{x \in A \mid \nu_{1}(x)=x\right\}$.

(2) $\left(\left[E_{r}(A)\right]^{[2]}, \wedge, \vee, \odot, \Rightarrow, \pi_{1}, \pi_{2},[\perp, \perp],[\perp, \top],[\top, \top]\right)$ is a right triangle algebra. 
Proof. (1) Let $\nu_{1}(x)=x$. Then $\nu_{1}(x)=\mu_{1}\left(\nu_{1}(x)\right)=\mu_{1}(x)$. Hence $\mu_{1}(x)=$ $x$. Let $\mu_{1}(x)=x$. Then $\mu_{1}(x)=\nu_{1}\left(\mu_{1}(x)\right)=\nu_{1}(x)$. Hence $\nu_{1}(x)=x$. Thus $\left\{x \in A \mid \nu_{1}(x)=x\right\}=\left\{x \in A \mid \nu_{1}(x)=x\right\}$.

(R1) For $x, y \in E_{r}(A), \nu_{1}(x \wedge y)=\nu_{1}(x) \wedge \nu_{1}(y)=x \wedge y$ and $\nu_{1}(x \vee y)=$ $\nu_{1}(x) \vee \nu_{1}(y)=x \vee y$. Then $x \wedge y, x \vee y \in E_{r}(A)$. Since $\nu_{1}(\perp)=\perp$ and $\nu_{1}(\perp)=\perp$, then $\perp, \top \in E_{r}(A)$.

Since $x \Rightarrow y=\nu_{1}(x) \Rightarrow \nu_{1}(y) \leq \nu_{1}\left(\nu_{1}(x) \Rightarrow \nu_{1}(y)\right) \leq \nu_{1}(x) \Rightarrow \nu_{1}(y)=x \Rightarrow$ $y$, then $x \Rightarrow y \in E_{r}(A)$. Since $y \leq x \Rightarrow x \otimes y$, we have $\nu_{1}(y) \leq \nu_{1}(x \Rightarrow x \otimes y)=$ $\nu_{1}(x) \Rightarrow \nu_{1}(x \otimes y)$. Hence $\nu_{1}(x) \otimes \nu_{1}(y) \leq \nu_{1}(x \otimes y)$. So, $x \otimes y=\nu_{1}(x) \otimes \nu_{1}(y) \leq$ $\nu_{1}(x \otimes y) \leq x \otimes y$, then $x \otimes y \in E_{r}(A)$.

(R2) For $x, y, z \in E_{r}(A),\left(\nu_{1}(x) \otimes \nu_{1}(y)\right) \otimes \nu_{1}(z)=(x \otimes y) \otimes z=x \otimes(y \otimes z)=$ $\nu_{1}(x) \otimes\left(\nu_{1}(y) \otimes \nu_{1}(z)\right)$ and $x \otimes \top=\nu_{1}(x) \otimes \nu_{1}(\top)=\nu_{1}(x) \otimes \top=\nu_{1}(x)=x$.

$(\mathrm{RR}) x \otimes y \leq z$ iff $\nu_{1}(x \otimes y)=\nu_{1}(x) \otimes \nu_{1}(y) \leq \nu_{1}(z)$ iff $\nu_{1}(y) \leq \nu_{1}(x) \Rightarrow$ $\nu_{1}(z)=\nu_{1}(x \Rightarrow z)$ iff $y \leq x \Rightarrow z$.

The following corollary is similarly proved that of Theorem 8 .

Corollary 9. Let $\left(A, \wedge, \vee, \otimes, \rightarrow, \nu_{2}, \mu_{2}, \perp, e_{2}, \top\right)$ be a left triangle lattice. Then the following properties hold.

(1) $\left(E_{l}(A), \wedge, \vee, \otimes, \rightarrow, \perp, \top\right)$ is a left residuated lattice where $E_{l}(A)=\{x \in$ $\left.A \mid \nu_{2}(x)=x\right\}=\left\{x \in A \mid \mu_{2}(x)=x\right\}$.

(2) $\left(\left[E_{l}(A)\right]^{[2]}, \wedge, \vee, \odot, \rightarrow, \pi_{1}, \pi_{2},[\perp, \perp],[\perp, \top],[\top, \top]\right)$ is a left triangle algebra.

Example 10. Let $K=\left\{(x, y) \in R^{2} \mid x>0\right\}$ be a set and we define an operation $\otimes: K \times K \rightarrow K$ as follows:

$$
\left(x_{1}, y_{1}\right) \otimes\left(x_{2}, y_{2}\right)=\left(x_{1} x_{2}, x_{1} y_{2}+y_{1}\right)
$$

Then $(K, \otimes)$ is a group with $e=(1,0),(x, y)^{-1}=\left(\frac{1}{x},-\frac{y}{x}\right)$.

We have a positive cone $P=\left\{(a, b) \in R^{2} \mid a=1, b \geq 0\right.$, or $\left.a>1\right\}$ because $P \cap P^{-1}=\{(1,0)\}, P \odot P \subset P,(a, b)^{-1} \odot P \odot(a, b)=P$ and $P \cup P^{-1}=K$. For $\left(x_{1}, y_{1}\right),\left(x_{2}, y_{2}\right) \in K$, we define

$$
\begin{aligned}
\left(x_{1}, y_{1}\right) \leq\left(x_{2}, y_{2}\right) & \Leftrightarrow\left(x_{1}, y_{1}\right)^{-1} \odot\left(x_{2}, y_{2}\right) \in P,\left(x_{2}, y_{2}\right) \odot\left(x_{1}, y_{1}\right)^{-1} \in P \\
& \Leftrightarrow x_{1}<x_{2} \text { or } x_{1}=x_{2}, y_{1} \leq y_{2} .
\end{aligned}
$$

Then $(K, \leq \otimes)$ is a lattice-group. The structure $\left(L, \odot, \Rightarrow, \rightarrow,\left(\frac{1}{2}, 1\right),(1,0)\right)$ is defined a lattice where $\perp=\left(\frac{1}{2}, 1\right)$ is the least element and $T=(1,0)$ is the 
greatest element from the following statements, for $\left(x_{1}, y_{1}\right)\left(x_{2}, y_{2}\right) \in L$,

$$
\begin{aligned}
& \left(x_{1}, y_{1}\right) \odot\left(x_{2}, y_{2}\right)=\left(x_{1}, y_{1}\right) \otimes\left(x_{2}, y_{2}\right) \vee\left(\frac{1}{2}, 1\right)=\left(x_{1} x_{2}, x_{1} y_{2}+y_{1}\right) \vee\left(\frac{1}{2}, 1\right) \\
& \left(x_{1}, y_{1}\right) \Rightarrow\left(x_{2}, y_{2}\right)=\left(\left(x_{1}, y_{1}\right)^{-1} \otimes\left(x_{2}, y_{2}\right)\right) \wedge(1,0)=\left(\frac{x_{2}}{x_{1}}, \frac{y_{2}-y_{1}}{x_{1}}\right) \wedge(1,0) \\
& \left(x_{1}, y_{1}\right) \rightarrow\left(x_{2}, y_{2}\right)=\left(\left(x_{2}, y_{2}\right) \otimes\left(x_{1}, y_{1}\right)^{-1}\right) \wedge(1,0)=\left(\frac{x_{2}}{x_{1}},-\frac{x_{2} y_{1}}{x_{1}}+y_{2}\right) \wedge(1,0) .
\end{aligned}
$$

(1) The structure $\left(L, \odot, \Rightarrow,\left(\frac{1}{2}, 1\right),(1,0)\right)$ is a right residuated lattice because $L$ is a totally order and the following statements:

$$
\begin{array}{ll}
\left(x_{1}, y_{1}\right) \vee\left(x_{2}, y_{2}\right)=\left(x_{2}, y_{2}\right) & \text { iff }\left(x_{1}, y_{1}\right) \leq\left(x_{2}, y_{2}\right) \\
\left(x_{1}, y_{1}\right) \wedge\left(x_{2}, y_{2}\right)=\left(x_{1}, y_{1}\right) & \text { iff }\left(x_{1}, y_{1}\right) \leq\left(x_{2}, y_{2}\right) \\
\left(x_{1}, y_{1}\right) \odot\left(x_{2}, y_{2}\right) \leq\left(x_{3}, y_{3}\right) & \text { iff }\left(x_{2}, y_{2}\right) \leq\left(\left(x_{1}, y_{1}\right)^{-1} \odot\left(x_{3}, y_{3}\right)\right) \wedge(1,0) \\
& \text { iff }\left(x_{2}, y_{2}\right) \leq\left(x_{1}, y_{1}\right) \Rightarrow\left(x_{3}, y_{3}\right) .
\end{array}
$$

We define

$$
\begin{gathered}
L^{[2]}=\left\{\left[\left(x_{1}, y_{1}\right),\left(x_{2}, y_{2}\right)\right] \mid\left(\left(x_{1}, y_{1}\right),\left(x_{2}, y_{2}\right)\right) \in L \times L,\left(x_{1}, y_{1}\right) \leq\left(x_{2}, y_{2}\right)\right\} \\
e_{1}=\left[\left(\frac{1}{2}, 1\right),(1,0)\right]=[\perp, \top], \\
{\left[\left(x_{1}, y_{1}\right),\left(x_{2}, y_{2}\right)\right] \wedge\left[\left(x_{3}, y_{3}\right),\left(x_{4}, y_{4}\right)\right]=\left[\left(x_{1}, y_{1}\right) \wedge\left(x_{3}, y_{3}\right),\left(x_{2}, y_{2}\right) \wedge\left(x_{4}, y_{4}\right)\right]} \\
{\left[\left(x_{1}, y_{1}\right),\left(x_{2}, y_{2}\right)\right] \vee\left[\left(x_{3}, y_{3}\right),\left(x_{4}, y_{4}\right)\right]=\left[\left(x_{1}, y_{1}\right) \vee\left(x_{3}, y_{3}\right),\left(x_{2}, y_{2}\right) \vee\left(x_{4}, y_{4}\right)\right]} \\
{\left[\left(x_{1}, y_{1}\right),\left(x_{2}, y_{2}\right)\right] \odot\left[\left(x_{3}, y_{3}\right),\left(x_{4}, y_{4}\right)\right]=\left[\left(x_{1}, y_{1}\right) \odot\left(x_{3}, y_{3}\right),\left(x_{2}, y_{2}\right) \odot\left(x_{4}, y_{4}\right)\right]} \\
{\left[\left(x_{1}, y_{1}\right),\left(x_{2}, y_{2}\right)\right] \Rightarrow\left[\left(x_{3}, y_{3}\right),\left(x_{4}, y_{4}\right)\right]} \\
=\left[\left(\left(x_{1}, y_{1}\right) \Rightarrow\left(x_{3}, y_{3}\right)\right) \wedge\left(\left(x_{2}, y_{2}\right) \Rightarrow\left(x_{4}, y_{4}\right)\right),\left(x_{2}, y_{2}\right) \Rightarrow\left(x_{4}, y_{4}\right)\right]
\end{gathered}
$$

Moreover, we define $\pi_{1}, \pi_{2}: L^{[2]} \rightarrow L^{[2]}$ as

$$
\begin{aligned}
& \pi_{1}\left(\left[\left(x_{1}, y_{1}\right),\left(x_{2}, y_{2}\right)\right]\right)=\left[\left(x_{1}, y_{1}\right),\left(x_{1}, y_{1}\right)\right] \\
& \pi_{2}\left(\left[\left(x_{1}, y_{1}\right),\left(x_{2}, y_{2}\right)\right]\right)=\left[\left(x_{2}, y_{2}\right),\left(x_{2}, y_{2}\right)\right] .
\end{aligned}
$$

Then $\left(L^{[2]}, \wedge, \vee, \odot, \Rightarrow, \pi_{1}, \pi_{2},[\perp, \perp], e_{1}=[\perp, \top],[\top, \top]\right)$ is a right triangle algebra from Theorem $6(3)$. Moreover, from Theorem $8,\left(E_{r}\left(L^{[2]}\right), \wedge, \vee, \otimes, \Rightarrow, \perp, \top\right)$ is a right residuated lattice where

$$
E_{r}\left(L^{[2]}\right)=\left\{\left[\left(x_{1}, y_{1}\right),\left(x_{1}, y_{1}\right)\right] \mid \pi_{1}\left(\left[\left(x_{1}, y_{1}\right),\left(x_{1}, y_{1}\right)\right]\right)=\left[\left(x_{1}, y_{1}\right),\left(x_{1}, y_{1}\right)\right]\right\} .
$$


(2) The structure $\left(L, \odot, \rightarrow,\left(\frac{1}{2}, 1\right),(1,0)\right)$ is a left residuated lattice because $L$ is a totally order and the following statements:

$$
\begin{aligned}
\left(x_{1}, y_{1}\right) \odot\left(x_{2}, y_{2}\right) \leq\left(x_{3}, y_{3}\right) \quad & \text { iff }\left(x_{1}, y_{1}\right) \leq\left(\left(x_{3}, y_{3}\right) \odot\left(\left(x_{1}, y_{1}\right)^{-1}\right)\right) \wedge(1,0) \\
& \text { iff }\left(x_{1}, y_{1}\right) \leq\left(x_{2}, y_{2}\right) \rightarrow\left(x_{3}, y_{3}\right) .
\end{aligned}
$$

We define

$$
\begin{aligned}
& L^{[2]}=\left\{\left[\left(x_{1}, y_{1}\right),\left(x_{2}, y_{2}\right)\right] \mid\left(\left(x_{1}, y_{1}\right),\left(x_{2}, y_{2}\right)\right) \in L \times L,\left(x_{1}, y_{1}\right) \leq\left(x_{2}, y_{2}\right)\right\} \\
& e=\left[\left(\frac{1}{2}, 1\right),(1,0)\right]=[\perp, \top], \\
& \quad\left[\left(x_{1}, y_{1}\right),\left(x_{2}, y_{2}\right)\right] \rightarrow\left[\left(x_{3}, y_{3}\right),\left(x_{4}, y_{4}\right)\right] \\
& =\left[\left(\left(\left(x_{1}, y_{1}\right) \rightarrow\left(x_{3}, y_{3}\right)\right) \wedge\left(\left(x_{2}, y_{2}\right) \rightarrow\left(x_{4}, y_{4}\right)\right),\left(x_{2}, y_{2}\right) \rightarrow\left(x_{4}, y_{4}\right)\right] .\right.
\end{aligned}
$$

Moreover, we define $\pi_{1}, \pi_{2}: L^{[2]} \rightarrow L^{[2]}$ as

$$
\begin{aligned}
& \pi_{1}\left(\left[\left(x_{1}, y_{1}\right),\left(x_{2}, y_{2}\right)\right]\right)=\left[\left(x_{1}, y_{1}\right),\left(x_{1}, y_{1}\right)\right] \\
& \pi_{2}\left(\left[\left(x_{1}, y_{1}\right),\left(x_{2}, y_{2}\right)\right]\right)=\left[\left(x_{2}, y_{2}\right),\left(x_{2}, y_{2}\right)\right] .
\end{aligned}
$$

Then $\left(L^{[2]}, \wedge, \vee, \odot, \rightarrow, \pi_{1}, \pi_{2},[\perp, \perp], e_{2}=[\perp, \top],[\top, \top]\right)$ is a left triangle algebra from Corollary 7. Moreover, from Corollary $9,\left(E_{l}\left(L^{[2]}\right), \wedge, \vee, \otimes, \rightarrow, \perp, \top\right)$ is a left residuated lattice where

$$
E_{l}\left(L^{[2]}\right)=\left\{\left[\left(x_{1}, y_{1}\right),\left(x_{1}, y_{1}\right)\right] \mid \pi_{1}\left(\left[\left(x_{1}, y_{1}\right),\left(x_{1}, y_{1}\right)\right]\right)=\left[\left(x_{1}, y_{1}\right),\left(x_{1}, y_{1}\right)\right]\right\}
$$

Theorem 11. Let $\left(A, \wedge, \vee, \otimes, \Rightarrow, \nu_{1}, \mu_{1}, \perp, e_{1}, \top\right)$ be a right triangle algebra and $R_{r}(A)=\left\{\left[\nu_{1}(x), \mu_{1}(x)\right] \mid x \in A\right\}$. Define

$$
\begin{aligned}
{\left[\nu_{1}(x), \mu_{1}(x)\right] \wedge\left[\nu_{1}(y), \mu_{1}(y)\right] } & =\left[\nu_{1}(x) \wedge \nu_{1}(y), \mu_{1}(x) \wedge \mu_{1}(y)\right], \\
{\left[\nu_{1}(x), \mu_{1}(x)\right] \vee\left[\nu_{1}(y), \mu_{1}(y)\right] } & =\left[\nu_{1}(x) \vee \nu_{1}(y), \mu_{1}(x) \vee \mu_{1}(y)\right], \\
{\left[\nu_{1}(x), \mu_{1}(x)\right] \odot\left[\nu_{1}(y), \mu_{1}(y)\right] } & =\left[\nu_{1}(x \otimes y), \mu_{1}(x \otimes y)\right], \\
{\left[\nu_{1}(x), \mu_{1}(x)\right] \Rightarrow\left[\nu_{1}(y), \mu_{1}(y)\right] } & =\left[\nu_{1}(x \Rightarrow y), \mu_{1}(x \Rightarrow y)\right], \\
\pi_{1}\left(\left[\nu_{1}(x), \mu_{1}(x)\right]\right)=\left[\nu_{1}(x), \nu_{1}(x)\right], & \pi_{2}\left(\left[\nu_{1}(x), \mu_{1}(x)\right]\right)=\left[\mu_{1}(x), \mu_{1}(x)\right] .
\end{aligned}
$$

Then we have the following properties:

(1) $x \leq y$ iff $\nu_{1}(x) \leq \nu_{1}(y)$ and $\mu_{1}(x) \leq \mu_{1}(y)$ iff $\left[\nu_{1}(x), \mu_{1}(x)\right] \leq\left[\nu_{1}(y), \mu_{1}(y)\right]$.

(2) $\left(R_{r}(A), \wedge, \vee, \odot, \Rightarrow, \pi_{1}, \pi_{2},[\perp, \perp],[\perp, \top],[\top, \top]\right)$ is a right triangle algebra with $\pi_{1}\left(\phi_{1}(x)\right)=\nu_{1}(x), \pi_{2}\left(\phi_{1}(x)\right)=\mu_{1}(x)$. 
(3) Define $\phi_{1}: A \rightarrow R_{r}(A)$ as $\phi_{1}(x)=\left[\nu_{1}(x), \mu_{1}(x)\right]$. Then $\phi_{1}$ is an isomorphism;i.e, $\phi_{1}$ is bijective,

$$
\begin{gathered}
\phi_{1}(x \wedge y)=\phi_{1}(x) \wedge \phi_{1}(y), \phi_{1}(x \vee y)=\phi_{1}(x) \vee \phi_{1}(y) \\
\phi_{1}(x \odot y)=\phi_{1}(x) \odot \phi_{1}(y), \phi_{1}(x \Rightarrow y)=\phi_{1}(x) \Rightarrow \phi_{1}(y) .
\end{gathered}
$$

Moreover, $\pi_{1}\left(\phi_{1}(x)\right)=\nu_{1}(x), \pi_{2}\left(\phi_{1}(x)\right)=\mu_{1}(x)$.

Proof. (1) First, we show that $x \leq y$ iff $\nu_{1}(x) \leq \nu_{1}(y)$ and $\mu_{1}(x) \leq \mu_{1}(y)$. Let $x \leq y$. Then $x \wedge y=x . \nu_{1}(x) \wedge \nu_{1}(y)=\nu_{1}(x)$ and $\mu_{1}(x) \wedge \mu_{1}(y)=\mu_{1}(x)$. Hence $\nu_{1}(x) \leq \nu_{1}(y)$ and $\mu_{1}(x) \leq \mu_{1}(y)$.

Conversely, since $\nu_{1}(x) \leq \nu_{1}(y)$ and $\mu_{1}(x) \leq \mu_{1}(y), \nu_{1}(x \wedge y)=\nu_{1}(x)$ and $\mu_{1}(x \wedge y)=\mu_{1}(x)$. By $(\mathrm{T} 5), x \wedge y=x$. Hence $x \leq y$.

Second, we show that $\nu_{1}(x) \leq \nu_{1}(y)$ and $\mu_{1}(x) \leq \mu_{1}(y)$ iff $\left[\nu_{1}(x), \mu_{1}(x)\right] \leq$ $\left[\nu_{1}(y), \mu_{1}(y)\right]$. Let $\nu_{1}(x) \leq \nu_{1}(y)$ and $\mu_{1}(x) \leq \mu_{1}(y)$. Then $\left[\nu_{1}(x), \mu_{1}(x)\right] \wedge$ $\left[\nu_{1}(y), \mu_{1}(y)\right]=\left[\nu_{1}(x), \mu_{1}(x)\right]$. Hence $\left[\nu_{1}(x), \mu_{1}(x)\right] \leq\left[\nu_{1}(y), \mu_{1}(y)\right]$. Conversely, it is similarly proved.

(2) (R1) Since $\left[\nu_{1}(\perp), \mu_{1}(\perp)\right]=[\perp, \perp]$ and $\left[\nu_{1}(\top), \mu_{1}(\top)\right]=[\top, T]$, then $[\perp, \perp]$ is the bottom element and $[\top, \top]$ is the top element.

(R2)

$$
\begin{aligned}
& \left(\left[\nu_{1}(x), \mu_{1}(x)\right] \odot\left[\nu_{1}(y), \mu_{1}(y)\right]\right) \odot\left[\nu_{1}(z), \mu_{1}(z)\right] \\
= & {\left[\nu_{1}(x \otimes y), \mu_{1}(x \otimes y)\right] \odot\left[\nu_{1}(z), \mu_{1}(z)\right] } \\
= & {\left[\nu_{1}((x \otimes y) \otimes z), \mu_{1}((x \otimes y) \otimes z)\right] } \\
= & {\left[\nu_{1}(x \otimes(y \otimes z)), \mu_{1}(x \otimes(y \otimes z))\right] } \\
= & {\left[\nu_{1}(x), \mu_{1}(x)\right] \odot\left(\left[\nu_{1}(y), \mu_{1}(y)\right] \odot\left[\nu_{1}(z), \mu_{1}(z)\right]\right) } \\
{\left[\nu_{1}(x), \mu_{1}(x)\right] \odot[\top, \top] } & =\left[\nu_{1}(x), \mu_{1}(x)\right] \odot\left[\nu_{1}(\top), \mu_{1}(\top)\right] \\
& =\left[\nu_{1}(x \otimes \top), \mu_{1}(x \otimes \top)\right]=\left[\nu_{1}(x), \mu_{1}(x)\right]
\end{aligned}
$$

$(\mathrm{RR})$

$$
\begin{aligned}
& {\left[\nu_{1}(x), \mu_{1}(x)\right] \odot\left[\nu_{1}(y), \mu_{1}(y)\right] \leq\left[\nu_{1}(z), \mu_{1}(z)\right]} \\
& \text { iff } \nu_{1}(x \otimes y) \leq \nu_{1}(z), \mu_{1}(x \otimes y) \leq \mu_{1}(z) \\
& \text { iff } \nu_{1}((x \otimes y) \vee z)=\nu_{1}(z), \mu_{1}((x \otimes y) \vee z)=\mu_{1}(z) \\
& \text { iff }(x \otimes y) \vee z=z, \text { iff }(x \otimes y) \leq z, \text { iff } y \leq x \Rightarrow z, \\
& \text { iff } \nu_{1}(y) \leq \nu_{1}(x \Rightarrow z), \mu_{1}(y) \leq \mu_{1}(x \Rightarrow z) \\
& \text { iff }\left[\nu_{1}(y), \mu_{1}(y)\right] \leq\left[\nu_{1}(x \Rightarrow z), \mu_{1}(x \Rightarrow z)\right] \\
& \text { iff }\left[\nu_{1}(y), \mu_{1}(y)\right] \leq\left[\nu_{1}(x), \mu_{1}(x)\right] \Rightarrow\left[\nu_{1}(z), \mu_{1}(z)\right]
\end{aligned}
$$




$$
\begin{aligned}
& \pi_{1}\left(\left[\nu_{1}(x), \mu_{1}(x)\right] \Rightarrow\left[\nu_{1}(y), \mu_{1}(y)\right]\right)=\left[\nu_{1}(x \Rightarrow y), \nu_{1}(x \Rightarrow y)\right] \\
& \pi_{1}\left(\left[\nu_{1}(x), \mu_{1}(x)\right]\right) \Rightarrow \pi_{1}\left(\left[\nu_{1}(y), \mu_{1}(y)\right]\right)=\left[\nu_{1}(x), \nu_{1}(x)\right] \Rightarrow\left[\nu_{1}(y), \nu_{1}(y)\right] \\
& =\left[\nu_{1}\left(\nu_{1}(x)\right), \mu_{1}\left(\nu_{1}(x)\right)\right] \Rightarrow\left[\nu_{1}\left(\nu_{1}(y)\right), \mu_{1}\left(\nu_{1}(y)\right)\right] \\
& =\left[\nu_{1}\left(\nu_{1}(x) \Rightarrow \nu_{1}(y)\right), \mu_{1}\left(\nu_{1}(x) \Rightarrow \nu_{1}(y)\right)\right]
\end{aligned}
$$

Since $\nu_{1}(x) \odot \nu_{1}(x \Rightarrow y) \leq \nu_{1}(x \odot(x \Rightarrow y)) \leq \nu_{1}(y)$, then $\nu_{1}(x \Rightarrow y) \leq \nu_{1}(x) \Rightarrow$ $\nu_{1}(y) \leq \nu_{1}\left(\nu_{1}(x) \Rightarrow \nu_{1}(y)\right)$ from $(\mathrm{R} 7)$. Moreover,

$$
\nu_{1}(x) \Rightarrow \nu_{1}(y) \leq \mu_{1}\left(\nu_{1}(x) \Rightarrow \nu_{1}(y)\right) \leq \mu_{1}\left(\nu_{1}(x)\right) \Rightarrow \mu_{1}\left(\nu_{1}(y)\right)=\nu_{1}(x) \Rightarrow \nu_{1}(y) .
$$

$(\mathrm{R} 5),(\mathrm{R} 7)$

$$
\begin{aligned}
& \pi_{1}\left(\left[\nu_{1}(x), \mu_{1}(x)\right] \Rightarrow\left[\nu_{1}(y), \mu_{1}(y)\right]\right)=\left[\nu_{1}(x \Rightarrow y), \nu_{1}(x \Rightarrow y)\right] \\
& \leq\left[\nu_{1}\left(\nu_{1}(x) \Rightarrow \nu_{1}(y)\right), \mu_{1}\left(\nu_{1}(x) \Rightarrow \nu_{1}(y)\right)\right] \\
& =\pi_{1}\left(\left[\nu_{1}(x), \mu_{1}(x)\right]\right) \Rightarrow \pi_{1}\left(\left[\nu_{1}(y), \mu_{1}(y)\right]\right) . \\
& \pi_{1}\left(\left[\nu_{1}(x), \mu_{1}(x)\right]\right) \Rightarrow \pi_{1}\left(\left[\nu_{1}(y), \mu_{1}(y)\right]\right) \\
& =\left[\nu_{1}\left(\nu_{1}(x) \Rightarrow \nu_{1}(y)\right), \mu_{1}\left(\nu_{1}(x) \Rightarrow \nu_{1}(y)\right)\right] \\
& =\left[\nu_{1}(x) \Rightarrow \nu_{1}(y), \nu_{1}(x) \Rightarrow \nu_{1}(y)\right] \\
& =\pi_{1}\left(\pi_{1}\left(\left[\nu_{1}(x), \mu_{1}(x)\right]\right) \Rightarrow \pi_{1}\left(\left[\nu_{1}(y), \mu_{1}(y)\right]\right)\right)
\end{aligned}
$$

(R6) Since $\nu_{1}\left(\mu_{1}(x)\right)=\mu_{1}(x), \mu_{1}(x), \mu_{1}(y) \in E_{r}(A)$. Then $\mu_{1}(x) \Rightarrow \mu_{1}(y) \in$ $E_{r}(A)$. Hence $\nu_{1}\left(\mu_{1}(x) \Rightarrow \mu_{1}(y)\right)=\mu_{1}(x) \Rightarrow \mu_{1}(y)$. Moreover,

$$
\begin{aligned}
& \nu_{1}\left(\mu_{1}(x) \Rightarrow \mu_{1}(y)\right)=\mu_{1}\left(\nu_{1}\left(\mu_{1}(x) \Rightarrow \mu_{1}(y)\right)\right)=\mu_{1}\left(\mu_{1}(x) \Rightarrow \mu_{1}(y)\right) . \\
& \pi_{2}\left(\left[\nu_{1}(x), \mu_{1}(x)\right]\right) \Rightarrow \pi_{2}\left(\left[\nu_{1}(y), \mu_{1}(y)\right]\right)=\left[\mu_{1}(x), \mu_{1}(x)\right] \Rightarrow\left[\mu_{1}(y), \mu_{1}(y)\right] \\
&=\left[\nu_{1}\left(\mu_{1}(x)\right), \mu_{1}\left(\mu_{1}(x)\right)\right] \Rightarrow\left[\nu_{1}\left(\mu_{1}(y)\right), \mu_{1}\left(\mu_{1}(y)\right)\right] \\
&=\left[\nu_{1}\left(\mu_{1}(x) \Rightarrow \mu_{1}(y)\right), \mu_{1}\left(\mu_{1}(x) \Rightarrow \mu_{1}(y)\right)\right] \\
&=\left[\mu_{1}(x) \Rightarrow \mu_{1}(y), \mu_{1}(x) \Rightarrow \mu_{1}(y)\right] . \\
& {\left[\nu_{1}(x), \mu_{1}(x)\right] \odot\left(\pi_{1}\left(\left[\nu_{1}(x), \mu_{1}(x)\right]\right) \Rightarrow \pi_{1}\left(\left[\nu_{1}(y), \mu_{1}(y)\right]\right)\right.} \\
& \odot\left(\pi_{2}\left(\left[\nu_{1}(x), \mu_{1}(x)\right]\right) \Rightarrow \pi_{2}\left(\left[\nu_{1}(y), \mu_{1}(y)\right]\right)\right) \\
&= {\left[\nu_{1}\left(\nu_{1}(x)\right), \mu_{1}\left(\mu_{1}(x)\right)\right] \odot\left(\left[\nu_{1}\left(\nu_{1}(x) \Rightarrow \nu_{1}(y)\right), \mu_{1}\left(\nu_{1}(x) \Rightarrow \nu_{1}(y)\right)\right]\right) } \\
& \odot\left(\left[\nu_{1}\left(\mu_{1}(x) \Rightarrow \mu_{1}(y)\right), \mu_{1}\left(\mu_{1}(x) \Rightarrow \mu_{1}(y)\right)\right]\right) \\
&=\left[\nu_{1}\left(\nu_{1}(x) \otimes\left(\nu_{1}(x) \Rightarrow \nu_{1}(y)\right) \otimes\left(\mu_{1}(x) \Rightarrow \mu_{1}(y)\right)\right),\right. \\
&\left.\mu_{1}\left(\mu_{1}(x) \otimes\left(\nu_{1}(x) \Rightarrow \nu_{1}(y)\right) \otimes\left(\mu_{1}(x) \Rightarrow \mu_{1}(y)\right)\right)\right] \\
& \leq\left[\nu_{1}\left(\nu_{1}(y)\right), \mu_{1}\left(\mu_{1}(y)\right)\right]=\left[\nu_{1}(y), \mu_{1}(y)\right]
\end{aligned}
$$

Hence 


$$
\begin{aligned}
\left(\pi_{1}\left(\left[\nu_{1}(x), \mu_{1}(x)\right]\right) \Rightarrow\right. & \pi_{1}\left(\left[\nu_{1}(y), \mu_{1}(y)\right]\right) \odot\left(\pi_{2}\left(\left[\nu_{1}(x), \mu_{1}(x)\right]\right)\right. \\
& \left.\Rightarrow \pi_{2}\left(\left[\nu_{1}(y), \mu_{1}(y)\right]\right)\right) \leq\left[\nu_{1}(x), \mu_{1}(x)\right] \Rightarrow\left[\nu_{1}(y), \mu_{1}(y)\right] .
\end{aligned}
$$

By a similar method, the condition (T6) holds. Other cases are easily proved.

(3) Define $\phi_{1}: A \rightarrow R_{r}(A)$ as $\phi_{1}(x)=\left[\nu_{1}(x), \mu_{1}(x)\right]$. Since $\nu_{1}(x) \leq \mu_{1}(x)$ and $\nu_{1}(x), \mu_{1}(x) \in E_{r}(X), \phi_{1}$ is well-defined. Moreover, $\phi_{1}(\perp)=\left[\nu_{1}(\perp), \mu_{1}(\perp)\right]$ $=[\perp, \perp], \phi_{1}\left(e_{1}\right)=\left[\nu_{1}\left(e_{1}\right), \mu_{1}\left(e_{1}\right)\right]=[\perp, \top]$ and $\phi_{1}(\top)=\left[\nu_{1}(\top), \mu_{1}(\top)\right]=$ $[\top, \top]$. If $\phi_{1}(x)=\phi_{1}(y)$, then $\nu_{1}(x)=\nu_{1}(y)$ and $\mu_{1}(x)=\mu_{1}(y)$. By (T5), $x=y$. Hence $\phi_{1}$ is injective.

For $\left[\nu_{1}(x), \mu_{1}(y)\right] \in R_{r}(A)$,

$$
\begin{aligned}
& \nu_{1}\left(\left(\nu_{1}(x) \vee e_{1}\right) \wedge \mu_{1}(x)\right)=\left(\nu_{1}\left(\nu_{1}(x)\right) \vee \nu_{1}\left(e_{1}\right)\right) \wedge \nu_{1}\left(\mu_{1}(x)\right) \\
& =\left(\nu_{1}(x) \vee \nu_{1}\left(e_{1}\right)\right) \wedge \nu_{1}\left(\mu_{1}(x)\right)=\nu_{1}(x) \wedge \mu_{1}(x)=\nu_{1}(x) \\
& \mu_{1}\left(\left(\nu_{1}(x) \vee e_{1}\right) \wedge \mu_{1}(x)\right)=\left(\mu_{1}\left(\nu_{1}(x)\right) \vee \mu_{1}\left(e_{1}\right)\right) \wedge \mu_{1}\left(\mu_{1}(x)\right) \\
& =\left(\mu_{1}(x) \vee \mu_{1}\left(e_{1}\right)\right) \wedge \mu_{1}\left(\mu_{1}(x)\right)=\top \wedge \mu_{1}(x)=\mu_{1}(x) .
\end{aligned}
$$

By $(\mathrm{T} 7),\left(\nu_{1}(x) \vee e_{1}\right) \wedge \mu_{1}(x)=x$. Similarly, $\nu_{1}(x) \vee\left(e_{1} \wedge \mu_{1}(x)\right)=x$. Moreover,

$$
\phi_{1}(x)=\phi_{1}\left(\left(\nu_{1}(x) \vee e_{1}\right) \wedge \mu_{1}(x)\right)=\phi_{1}\left(\nu_{1}(x) \vee\left(e_{1} \wedge \mu_{1}(x)\right)\right)=\left[\nu_{1}(x), \mu_{1}(y)\right] .
$$

Hence $\phi_{1}$ is surjective.

$$
\begin{aligned}
\nu_{1}(x \otimes y) & =\nu_{1}\left(\left(\nu_{1}(x) \vee\left(e_{1} \wedge \mu_{1}(x)\right) \otimes\left(\nu_{1}(y) \vee\left(e_{1} \wedge \mu_{1}(y)\right)\right)\right.\right. \\
\mu_{1}(x \otimes y)= & \mu_{1}\left(\left(\nu_{1}(x) \vee\left(e_{1} \wedge \mu_{1}(x)\right) \otimes\left(\nu_{1}(y) \vee\left(e_{1} \wedge \mu_{1}(y)\right)\right)\right.\right. \\
\phi_{1}(x \otimes y)= & {\left[\nu_{1}(x \otimes y), \mu_{1}(x \otimes y)\right] } \\
= & {\left[\nu _ { 1 } \left(\left(\nu_{1}(x) \vee\left(e_{1} \wedge \mu_{1}(x)\right) \otimes\left(\nu_{1}(y) \vee\left(e_{1} \wedge \mu_{1}(y)\right)\right),\right.\right.\right.} \\
& \mu_{1}\left(\left(\nu_{1}(x) \vee\left(e_{1} \wedge \mu_{1}(x)\right) \otimes\left(\nu_{1}(y) \vee\left(e_{1} \wedge \mu_{1}(y)\right)\right)\right]\right. \\
= & {\left[\nu_{1}(x), \mu_{1}(x)\right] \odot\left[\nu_{1}(y), \mu_{1}(y)\right] } \\
= & \phi_{1}(x) \odot \phi_{1}(y) \\
= & {\left[\nu_{1}(x \Rightarrow y), \mu_{1}(x \Rightarrow y)\right] } \\
= & {\left[\nu _ { 1 } \left(\left(\nu_{1}(x) \vee\left(e_{1} \wedge \mu_{1}(x)\right) \Rightarrow\left(\nu_{1}(y) \vee\left(e_{1} \wedge \mu_{1}(y)\right)\right),\right.\right.\right.} \\
& \mu_{1}\left(\left(\nu_{1}(x) \vee\left(e_{1} \wedge \mu_{1}(x)\right) \Rightarrow\left(\nu_{1}(y) \vee\left(e_{1} \wedge \mu_{1}(y)\right)\right)\right]\right. \\
= & {\left[\nu_{1}(x), \mu_{1}(x)\right] \Rightarrow\left[\nu_{1}(y), \mu_{1}(y)\right] } \\
= & \phi_{1}(x) \Rightarrow \phi_{1}(y)
\end{aligned}
$$

From Theorem 11, we similarly prove the following corollary.

Corollary 12. Let $\left(A, \wedge, \vee, \otimes, \rightarrow, \nu_{2}, \mu_{2}, \perp, e_{2}, \top\right)$ be a left triangle algebra and $R_{l}(A)=\left\{\left[\nu_{2}(x), \mu_{2}(x)\right] \mid x \in A\right\}$. Define

$$
\begin{aligned}
{\left[\nu_{2}(x), \mu_{2}(x)\right] \wedge\left[\nu_{2}(y), \mu_{2}(y)\right] } & =\left[\nu_{2}(x) \wedge \nu_{2}(y), \mu_{2}(x) \wedge \mu_{2}(y)\right], \\
{\left[\nu_{2}(x), \mu_{2}(x)\right] \vee\left[\nu_{2}(y), \mu_{2}(y)\right] } & =\left[\nu_{2}(x) \vee \nu_{2}(y), \mu_{2}(x) \vee \mu_{2}(y)\right], \\
{\left[\nu_{2}(x), \mu_{2}(x)\right] \odot\left[\nu_{2}(y), \mu_{2}(y)\right] } & =\left[\nu_{2}(x \otimes y), \mu_{2}(x \otimes y)\right], \\
{\left[\nu_{2}(x), \mu_{2}(x)\right] \rightarrow\left[\nu_{2}(y), \mu_{2}(y)\right] } & =\left[\nu_{2}(x \rightarrow y), \mu_{2}(x \rightarrow y)\right],
\end{aligned}
$$




$$
\pi_{1}\left(\left[\nu_{2}(x), \mu_{2}(x)\right]\right)=\left[\nu_{2}(x), \nu_{2}(x)\right], \pi_{2}\left(\left[\nu_{2}(x), \mu_{2}(x)\right]\right)=\left[\mu_{2}(x), \mu_{2}(x)\right] .
$$

Then we have the following properties.

(1) $x \leq y$ iff $\nu_{2}(x) \leq \nu_{2}(y)$ and $\mu_{2}(x) \leq \mu_{2}(y)$ iff $\left[\nu_{2}(x), \mu_{2}(x)\right] \leq\left[\nu_{2}(y), \mu_{2}(y)\right]$.

(2) $\left(R_{l}(A), \wedge, \vee, \odot, \Rightarrow, \pi_{1}, \pi_{2},[\perp, \perp],[\perp, \top],[\top, \top]\right)$ is a left triangle algebra.

(3) Define $\phi_{2}: A \rightarrow R_{l}(A)$ as $\phi_{2}(x)=\left[\nu_{2}(x), \mu_{2}(x)\right]$. Then $\phi_{2}$ is an isomorphism;i.e, $\phi_{2}$ is bijective,

$$
\begin{gathered}
\phi_{2}(x \wedge y)=\phi_{2}(x) \wedge \phi_{2}(y), \phi_{2}(x \vee y)=\phi_{2}(x) \vee \phi_{2}(y) \\
\phi_{2}(x \odot y)=\phi_{2}(x) \odot \phi_{2}(y), \phi_{2}(x \rightarrow y)=\phi_{2}(x) \rightarrow \phi_{2}(y) .
\end{gathered}
$$

\section{References}

[1] C. Alcalde, A. Burusco, R. Fuentes-Gonzalez, A constructive method for the definition of interval-valued fuzzy implication operators, Fuzzy Sets and Systems, 153 (2005), 211-227.

[2] R. Bělohlávek, Concept lattices and order in fuzzy logic, Ann. Pure Appl. Logic, 128 (2004),277-298.

[3] G. Deschrijver, Triangular norms which are meet-morphisms in intervalvalued fuzzy set theory, Fuzzy Sets and Systems, 181 (2011), 88-101.

[4] G. Deschrijver, Charaterizations of (weakly) Archimedean t-norms in interval-valued fuzzy set theory, Fuzzy Sets and Systems, 160 (2009), 30803102 .

[5] G. Deschrijver, A representation of t-norms in interval-valued fuzzy set theory, Fuzzy Sets and Systems, 159 (2008), 1597-1618.

[6] G. Georgescu, A. Popescue, Non-commutative Galois connections, Soft Computing, 7 (2003), 458-467.

[7] G. Georgescu, A. Popescue, Non-dual fuzzy connections, Arch. Math. Log. 43 (2004), 1009-1039.

[8] P. Hájek, Metamathematices of Fuzzy Logic, Kluwer Academic Publishers, Dordrecht (1998). 
[9] U. Höhle, E.P. Klement, Non-Classical Logic and their Applications to Fuzzy Subsets, Kluwer Academic Publisher, Boston (1995).

[10] Z. Pawlak, Rough sets, Int. J. Comput. Inf. Sci., 11 (1982), 341-356.

[11] Z. Pawlak, Rough probability, Bull. Pol. Acad. Sci. Math., 32 (1984), 607615.

[12] B. Van Gasse, C. Cornelis, G. Deschrijver, E.E. Kerre, Triangle algebras, Fuzzy Sets and Systems, 159 (2008), 1042-1060.

[13] B. Van Gasse, C. Cornelis, G. Deschrijver, E.E. Kerre, A characterization of interval-valued residuated lattices, Int. J. Approxi. Reason, 49 (2008), $478-487$.

[14] E. Turunen, Mathematics Behind Fuzzy Logic, Springer-Verlag (1999). 
